\title{
Load Sensitive Power Saving Technique for 4G Mobile Network under Limited User Traffic
}

\author{
Mohammad Asif Hossain', Mohammad Imdadul Islam², Mohamed Ruhul Amin ${ }^{1}$ \\ ${ }^{1}$ Department of Electronics and Communications Engineering, East West University, Dhaka, Bangladesh \\ ${ }^{2}$ Department of Computer Science and Engineering, Jahangirnagar University, Savar, Bangladesh \\ Email: ramin@ewubd.edu
}

Received 18 February 2016; accepted 9 May 2016; published 12 May 2016

Copyright (C) 2016 by authors and Scientific Research Publishing Inc.

This work is licensed under the Creative Commons Attribution International License (CC BY).

http://creativecommons.org/licenses/by/4.0/

(c) (i) Open Access

\begin{abstract}
Power saving is one of the key factors of emerging 4G mobile network as well as in IEEE 802.16e wireless metropolitan area networks (Wireless MAN). An efficient power saving mechanism is the heart for the guarantee of a long operating lifetime for a mobile subscriber station (MS), because MSs are normally driven by rechargeable batteries. It is a vital factor for Base Station (BS) of the same network. One of the most important features of $5 \mathrm{G}$ mobile is the extension of battery energy 10 times greater than the present days. In this context, the evaluation of duration of sleep mode of BS or MS based on traffic load of a network is now a new era of research work. In this paper, such analysis has been done based on two statistical models: Poisson's pdf and Engset pdf. The concept of complete sharing and partitioning of user group of teletraffic engineering is applied to measure the possibility of getting leisure time of BS or MS. Both the traffic models used in the paper are applicable in both limited and unlimited user network, i.e. in micro and fem to cellular network of 4G and 5G.
\end{abstract}

\section{Keywords}

Markovian Chain, Sleep Mode, Packet Arrival Rate, Schedulability and Probability of Data Accumulation

\section{Introduction}

Nowadays, wireless communications play an essential role in our daily life. People's connections with their society, culture and even one another are about to change due to wireless communications' ease availability and newer and faster mobile devices. The fourth generation (4G) of wireless standards has been specified by the In- 
ternational Telecommunications Union to meet the peak speed requirement of 1 Gbps for stationary connections and $100 \mathrm{Mbps}$ for a mobile connection [1]. The 4G service is designed to offer a faster and secured all-IP, roaming mobile broadband solution. The user can experience high speed network applications, including on-demand High Definition IP Television (HD IP-TV), Voice/Video-over-IP services, on-demand gaming, and high-speed internet access by using Smartphone. But the main challenge will remain with the power consumption of both the Smartphone and the BS.

All types of traffic in wired and wireless network have the property of ON-OFF governed by the behavior of users. For example, HTTP traffic of web browsing, FTP traffic between two computers, E-mail traffic among users or mail-server to users, voice traffic of telephony or mobile communications and multimedia traffic of 3G and 4G mobile communications have ON-OFF state. Each of the above traffic is modeled by two-state Markov chain; where one state is ON, i.e. there are some traffic which make the channel in serving condition and in OFF state i.e. there is not traffic hence the devices are in sleep mode. In [2], a new energy saving mechanism is proposed where sleep interval is changed considering traffic rate for IEEE $802.16 \mathrm{e} / \mathrm{m}$. The energy consumption is plotted against packet arrival rate for the case of analytical and simulation work. Similar analysis is found in [3] with statistical model of traffic. In [4], the authors' emphasis on a specific parameter: "unavailability interval ratio" for three different types of traffic. The traffics are: Best Effort (BE) and Non-Real-Time Variable Rate (NRT-VR) type (type I), Unsolicited Grant Service (UGS) and Real-Time Variable Rate (RTVR) type (type II) and for multicast connections as well as for management services (type III). The profile of "unavailability interval ratio" against time is shown for three different types of traffic. In [5], the power consumption of Evolve Node of LTE (Long Term Evolution) is proposed based on Discontinuous Transmission (DTX) mechanism, i.e. the node B is switched off when there is no packet to be served. The average power consumption and latency are measured by two methods: arithmetic average (AA) and another is the adaptive exponentially smoothed average (AeSA). The profile of power consumption and latency is shown against average inter-arrival time for both the methods. Another mechanism of energy saving is Discontinuous Reception Mechanism (DRX) which has been analyzed in [6]. DRX is to allow MS turning off their radio interfaces and going to sleep to save energy when no data needs to be received.

The size of sleep window is related with current traffic load for IEEE 802.16e in [7] and similar concept is applied in [8] by the same authors for LTE. The traffic arrival process is considered to follow Poisson's distribution in the paper. Power saving can be done comfortably when both UL and DL traffic are off. If UL and DL traffic are asynchronous then UL/DL traffic is delayed to make them synchronize with sleep interval. Such concept is applied in [9]; where authors plot the energy consumption against arrival rate of UL and DL. In this paper, limited user traffic model called PCT-II (Pure Chance Traffic II) for a subscriber station (SS) which is sensitive to offered traffic of the user group is dealt with. PCT I \& PCT II both are a type of traffic in which we assume that the service times are exponentially distributed. But the arrival process in PCT I is a Poisson process while in PCT II is formed by the limited number of sources. PCT I is also known as Erlang traffic while PCT II is known as Engset traffic [10].

The entire paper is organized as: section 2 deals with both limited and unlimited user's traffic to determine probability of data accumulation, section 3 provides the results based on statistical analysis of section 2 and section 4 concludes the entire analysis.

\section{Traffic Model}

\subsection{Unlimited Source Traffic Model}

Let $T$ is the length of TS (time slot) or frame for packet transmission. If $\lambda$ is the arrival rate of a packet for a particular user then the probability of arrival of $i$ packets over a duration of $[0, T]$ is expressed by Poisson's pdf like [11] [12]:

$$
p_{i}=\frac{(\lambda T)^{i} \mathrm{e}^{-\lambda T}}{i !} \text {. }
$$

If an observation time of $k$ slots is taken and $j$ packets arrives over [0, $k T]$, then its probability,

$$
p_{j}=\frac{(\lambda k T)^{j} \mathrm{e}^{-\lambda k T}}{j !} .
$$


If the packet number $N_{t h}$ is considered as the number of threshold packets, then the probability of arriving a number of packets greater than $N_{t h}$ over duration of $k$ frame is expressed as:

$$
\begin{aligned}
p\left(k, N_{t h}\right) & =\sum_{i=N_{t h}+1}^{\infty} \frac{(\lambda k T)^{i} \mathrm{e}^{-\lambda k T}}{i !} \\
& =1-\sum_{i=0}^{N_{t h}} \frac{(\lambda k T)^{i} \mathrm{e}^{-\lambda k T}}{i !} .
\end{aligned}
$$

According to [6],

The length of one awake-and-sleep cycle $=k^{*}=\min \left\{k \mid p\left(k, N_{t h}\right) \geq P_{t h \_}\right\}$.

The graphical presentation of Equation (4) is shown in Figure 1.

Let us consider, a subscriber station $i$ of $m$ users under a BS. The average arrival rate of packet of individual user are $\lambda_{1}, \lambda_{2}, \cdots, \lambda_{\mathrm{m}}$. and the corresponding length of one awake-and-sleep cycle are: $k_{1}^{*}, k_{2}^{*}, \cdots, k_{m}^{*}$.

Taking, $k_{i}^{\#}=2^{\log _{2}\left\lfloor k_{i}^{*}\right\rfloor}$, if $\sum_{i=1}^{m} \frac{1}{k_{i}^{\#}}<1$, then BS will have the opportunity to enter in sleep mode.

\subsection{Limited Source Traffic Model}

Let us now concentrate on the traffic model of limited user which is consistent with a SS (Sample Space). Figure 2 represents the network of limited user; where the traffic parameters are:

$$
\begin{aligned}
& N \rightarrow \text { number of users, } \\
& \lambda \rightarrow \text { average packet arrival rate/user, } \\
& \mu \rightarrow \text { packet termination rate, } \\
& M \rightarrow \text { Peak information rate, } \\
& m \rightarrow \text { average bit rate of individual user. }
\end{aligned}
$$

Therefore the number of traffic channel, $n=\frac{M}{m}$.

The traffic model of Figure 2 can be represented by Markov chain like Figure 3; where all the traffic parameters are mentioned before.

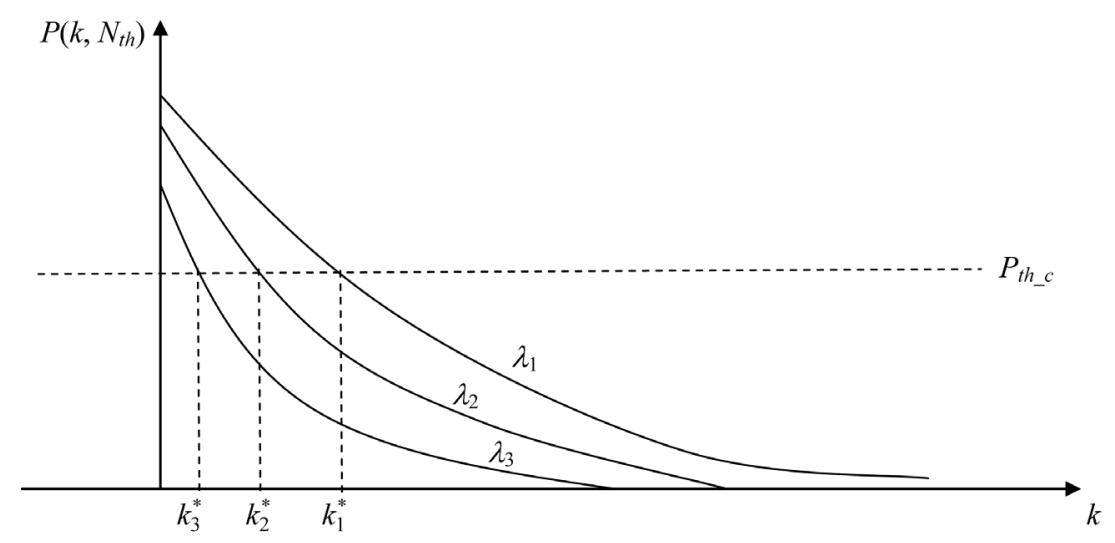

Figure 1. The graphical solution of Equation (4).

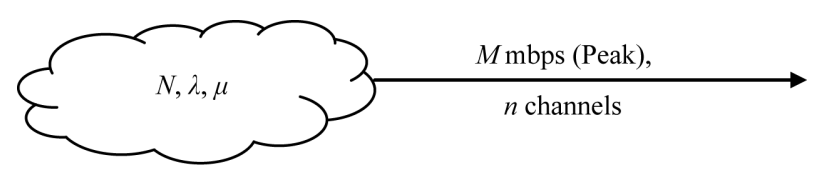

Figure 2. Traffic model of ith SS. 


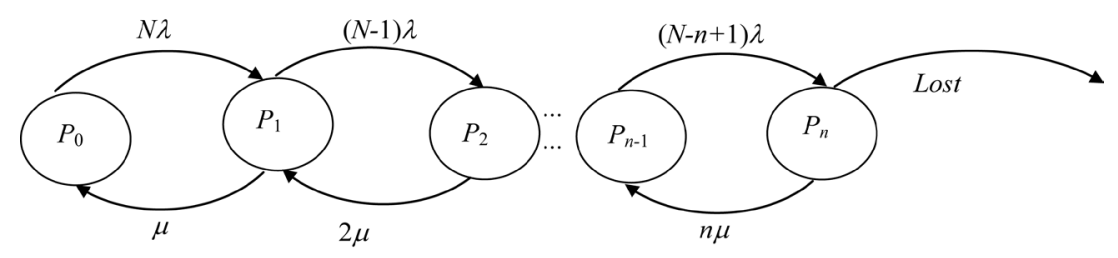

Figure 3. State transition chain of limited user.

Applying cut equations [13]-[15] between nodes,

$$
\begin{gathered}
P_{0} N \lambda=P_{1} \mu \Rightarrow P_{1}=P_{0} N(\lambda / \mu) \\
P_{1}(N-1) \lambda=P_{2} 2 \mu \Rightarrow P_{2}=P_{1}(N-1) \lambda / 2 \mu=P_{0} N(N-1)(\lambda / \mu)^{2}=P_{0}\left(\begin{array}{c}
N \\
2
\end{array}\right)(\lambda / \mu)^{2} .
\end{gathered}
$$

Similarly,

$$
P_{x}=P_{0}\left(\begin{array}{c}
N \\
x
\end{array}\right)(\lambda / \mu)^{x}
$$

where, $\left(\begin{array}{l}N \\ n\end{array}\right)=\frac{N !}{n !(N-n) !}$.

Now for entire sample space,

$$
\begin{aligned}
& P_{0}+P_{1}+P_{2}+P_{3}+\cdots+P_{n}=1 \\
& \Rightarrow P_{0}\left\{1+N\left(\frac{\lambda}{\mu}\right)+\left(\begin{array}{c}
N \\
2
\end{array}\right)\left(\frac{\lambda}{\mu}\right)^{2}+\left(\begin{array}{c}
N \\
3
\end{array}\right)\left(\frac{\lambda}{\mu}\right)^{3}+\cdots+\left(\begin{array}{c}
N \\
n
\end{array}\right)\left(\frac{\lambda}{\mu}\right)^{n}\right\}=1 \\
& \Rightarrow P_{0}=\frac{1}{\sum_{i=0}^{n}\left(\begin{array}{c}
N \\
i
\end{array}\right)\left(\frac{\lambda}{\mu}\right)^{i}}
\end{aligned}
$$

$\therefore$ The normalized probability state,

$$
P_{x}=\frac{\left(\begin{array}{c}
N \\
x
\end{array}\right)\left(\frac{\lambda}{\mu}\right)^{x}}{\sum_{i=0}^{n}\left(\begin{array}{c}
N \\
i
\end{array}\right)\left(\frac{\lambda}{\mu}\right)^{i}} .
$$

The blocking probability is the probability of occupancy of all traffic channels is,

$$
B(N, \lambda, n)=\frac{\left(\begin{array}{c}
N \\
n
\end{array}\right)\left(\frac{\lambda}{\mu}\right)^{n}}{\sum_{i=0}^{n}\left(\begin{array}{c}
N \\
i
\end{array}\right)\left(\frac{\lambda}{\mu}\right)^{i}} .
$$

Let us determine $K_{i}^{*}$ of Equation (4) and $K_{i}^{\#}$ of the $\mathrm{SS}_{i .}$.

First of all we will evaluate, $\sum_{i=1}^{N} \frac{1}{K_{i}^{\#}}=S$.

If $S>1$, it then splits the users of ith SS or combines users of different SSs.

\section{Result}

This section deals with both analytical and simulation results on "probability of data accumulation". The analytical part of this section is done by MATHCAD 14 and the simulation work is done in Matlab 13. First of all we 
discuss about Poisson's traffic in determining the profile of probability of data accumulation. Taking $T=2 \mathrm{~ms}$ and $N_{t h}=40$ and the variation of probability of data accumulation against $K$ is shown in Figure 4 where the packet arrival rate $\lambda$ (packets/ms) is taken as a parameter. Here we consider six user groups of different packet arrival rate shown in the legend of the graph. The threshold value of probability data accumulation depends on the offered traffic of a SS (or BS), usually derived from long observation time of the network. In this paper we take it 0.2 (as an example) shown by horizontal line cross symbols. For heavily loaded network this value is far greater than 0.2 where as for a lightly loaded network it is much smaller than 0.2 . From the intersection of the horizontal threshold line and the six curves of different $\lambda$ provides the minimum value of $K$ represented as $K^{*}$ in [16]. The numerical value of $K^{\#}$ i.e. $2^{\left\lfloor\log _{2} K^{*}\right\rfloor}$ are found as: $K_{1}^{\#}=8, K_{2}^{\#}=8, K_{3}^{\#}=8, K_{4}^{\#}=8, K_{5}^{\#}=16$ and $K_{6}^{\#}=16$. The schedulability, $\sum_{i}\left(1 / K_{i}^{\#}\right)=0.625<1$ hence the BS has the scope of entering sleep mode. If the threshold probability is lowered to 0.05 then the schedulability, $\sum_{i}\left(1 / K_{i}^{\#}\right)=0.688<1$, still the BS has the chance to enter in sleeping mode.

Now the user group-1 and 2 are combined together and the arrival rate of the combined group becomes 2.6 packets/s. The value of $K^{\#}$ are found as: $K_{1}^{\#}=8, K_{2}^{\#}=8, K_{3}^{\#}=8, K_{4}^{\#}=8$ and $K_{5}^{\#}=8$. The schedulability $\sum_{i}\left(1 / K_{1}^{\#}\right)=0.625<1$ hence the BS still has the scope of entering sleep mode as shown in Figure 5 Combining group 1,2 and 3 we get $K_{1}^{\#}=4, K_{2}^{\#}=8, K_{3}^{\#}=8$ and $K_{4}^{\#}=8$. The schedulability $\sum_{i}\left(1 / K_{i}^{\#}\right)=0.625<1$, therefore again the BS has the scope of entering sleep mode. If the probability of threshold is lowered to 0.05 then $K_{1}^{\#}=2, K_{2}^{\#}=4, K_{3}^{\#}=4$ and $K_{4}^{\#}=4$, then $\sum_{i}\left(1 / K_{i}^{\#}\right)=1.24>1$ i.e. beyond the safe zone. Finally combining group 1,2 and 3 and also using another combination of group 4 and 5 , we get schedulability $\sum_{i}\left(1 / K_{i}^{\#}\right)=1 / 4+1 / 4+1 / 8=0.625<1$. If the arrival rate is increased to $1.6,1.8,2,2.2,2.4$ and 2.6 , then schedulability $\sum_{i}\left(1 / K_{i}^{\#}\right)=6 / 8=0.75$, but still less than unity. The combination of any group will provide the result of success. If the arrival rate is increased like Figure 5 then result is found as: $K_{1}^{\#}=4, K_{2}^{\#}=4, K_{3}^{\#}=4$, $K_{4}^{\#}=8, \quad K_{5}^{\#}=8$ and $K_{6}^{\#}=8$. The schedulability $\sum_{i}\left(1 / K_{i}^{\#}\right)=1.125>1$ hence the BS does not have any scope of entering sleep mode. Combination of any group will provide results of failure. For example if group 1 and 2 are combined then result will be: $K_{1}^{\#}=4, K_{2}^{\#}=4, K_{3}^{\#}=4, K_{4}^{\#}=8$ and $K_{5}^{\#}=8$. The schedulability $\sum_{i}\left(1 / K_{i}^{\#}\right)=1.125>1$ hence again the BS does not have any scope of entering sleep mode. The entire analysis is done based on Poisson's model where result does not reveal any reflection of number of users.

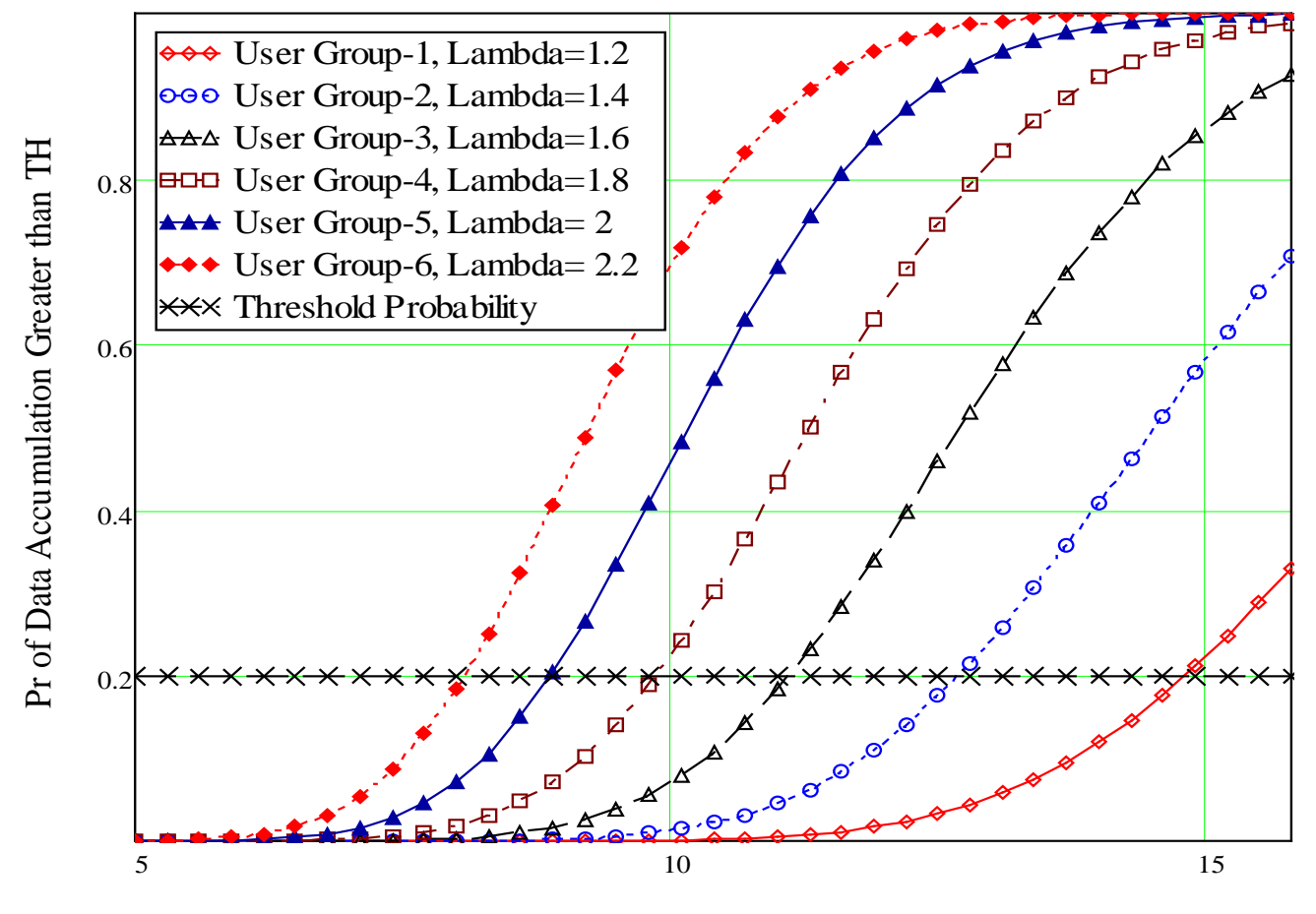

$\mathrm{K}$

Figure 4. Variation of probability of data accumulation. 


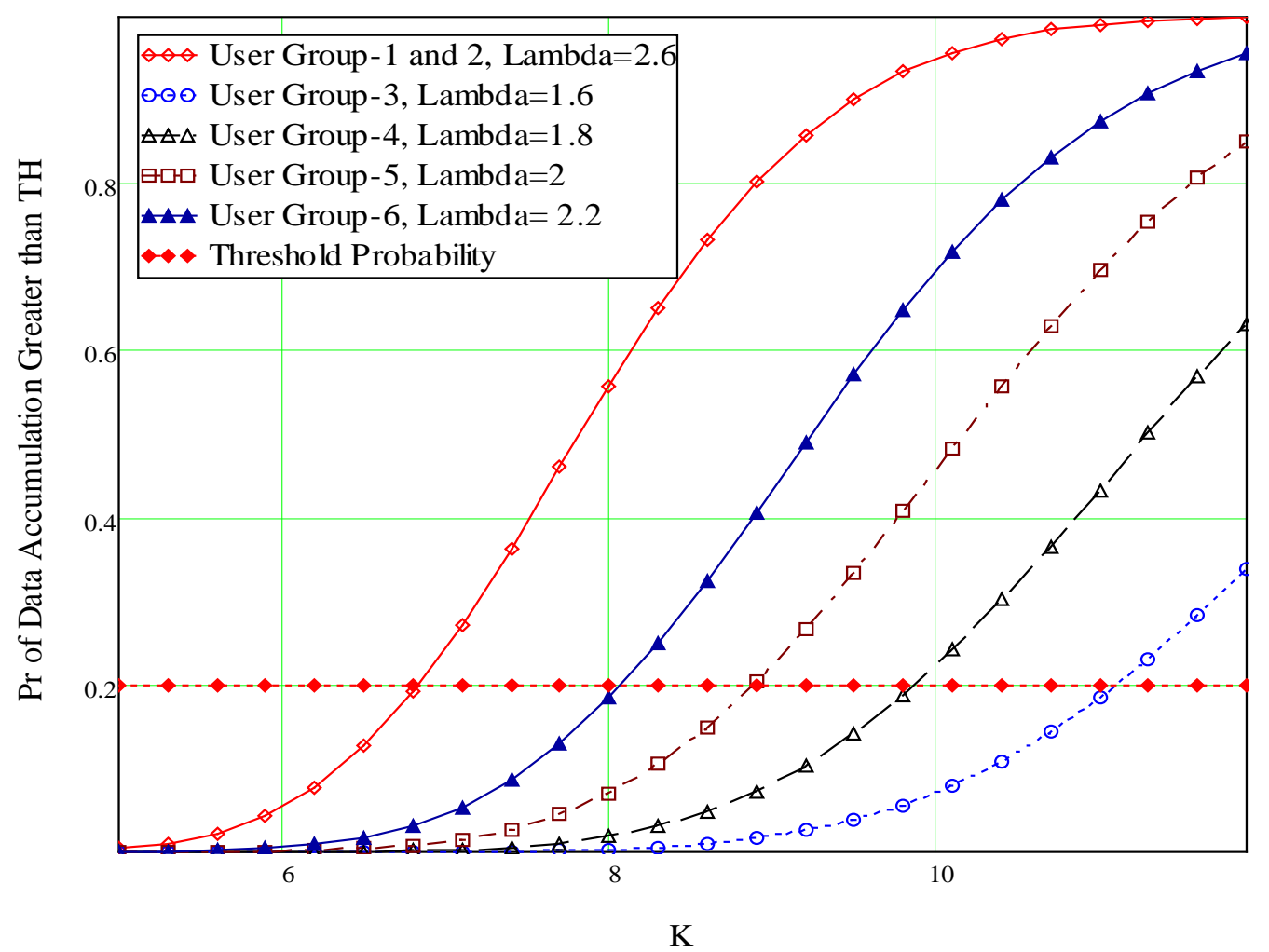

Figure 5. Combining user group 1 and 2.

Next part of the result section deals with traffic of limited user group called Engset traffic model. Instead of call arrival rate of Erlang's traffic (PCT-I traffic), here we have to consider packet arrival rate per user as shown in the legend of graphs of Figures 6-8. Taking: $N=50, n=35$ and $T=2$ we get the profile of probability of data accumulation taking arrival rate per user as a parameter shown in Figure 7. In this case schedulability, $\sum_{i}\left(1 / K_{i}^{\#}\right)=1 / 4+1 / 8+1 / 8+1 / 8+1 / 16+1 / 16=0.875<1$. Combining group 1 and $2 ; 3$ and 4 provides the profile shown in Figure 8 . Now the schedulability, $\sum_{i}\left(1 / K_{i}^{\#}\right)=1 / 2+1 / 4+1 / 4+1 / 8=1.125>1$. Therefore combination or separation of users group is a vital factor for Engset model to get sleeping slot of BS.

Finally we apply Monte-Carlo simulation on 10,000 random variables following exponential pdf (since we consider $\mathrm{M} / \mathrm{M} / \mathrm{n} / \mathrm{K}$ traffic where interarrival and service time follow exponential pdf) we evaluate the profile of "probability of data accumulation greater than threshold" against $K$ shown in Figure 9. The simulation results are found much closed to analytical model and reveals the confidence level of $98 \%$. Taking $K^{\#}=2^{\left\lfloor\log _{2} K^{*}\right\rfloor}$, our analytical and simulation results are found identical, since simulation results resembles to analytical case with less than $2 \%$ error. In this paper we mainly deal with the parameter schedulability to determine the probability of a BS (BS for uplink and SS for downlink) to enter in sleep cycle. Once the probability of entering sleep mode $P_{S}$ is determined, we can determine the portion of time the SS (or BS) enters in sleep mode hence we can get the "power saving factor" of [17]; where the paper shows the profile of "power saving factor" against the length of sleep cycle. If the energy consumption of a SS per unit time is $E_{L}$ during listening window and that of sleep cycle is $E_{S}$ then the consumed energy will be $\left\{\left(1-P_{S}\right) E_{L}+P_{S} E_{S}\right\} T$; where $T$ is the observation time of the SS.

\section{Conclusion}

In this paper, the probability of getting opportunity of a BS entering in sleeping mode is determined based on Poisson's and Engset traffic model considering arrival rate of aggregate traffic of a mobile cellular network. Very close results of the simulation with the analytical model have been found in this paper. Still, there are more scopes to work on the new originating and handoff traffic separately with suitable call admission scheme to observe the individual impact on entering leisure/sleep mode of a BS. In 3G mobiles, both circuit and packet switch exist at radio and core network but $4 \mathrm{G}$ emphasizes on packet switching. That is why $\mathrm{M} / \mathrm{G} / \mathrm{n} / \mathrm{K}$ traffic 


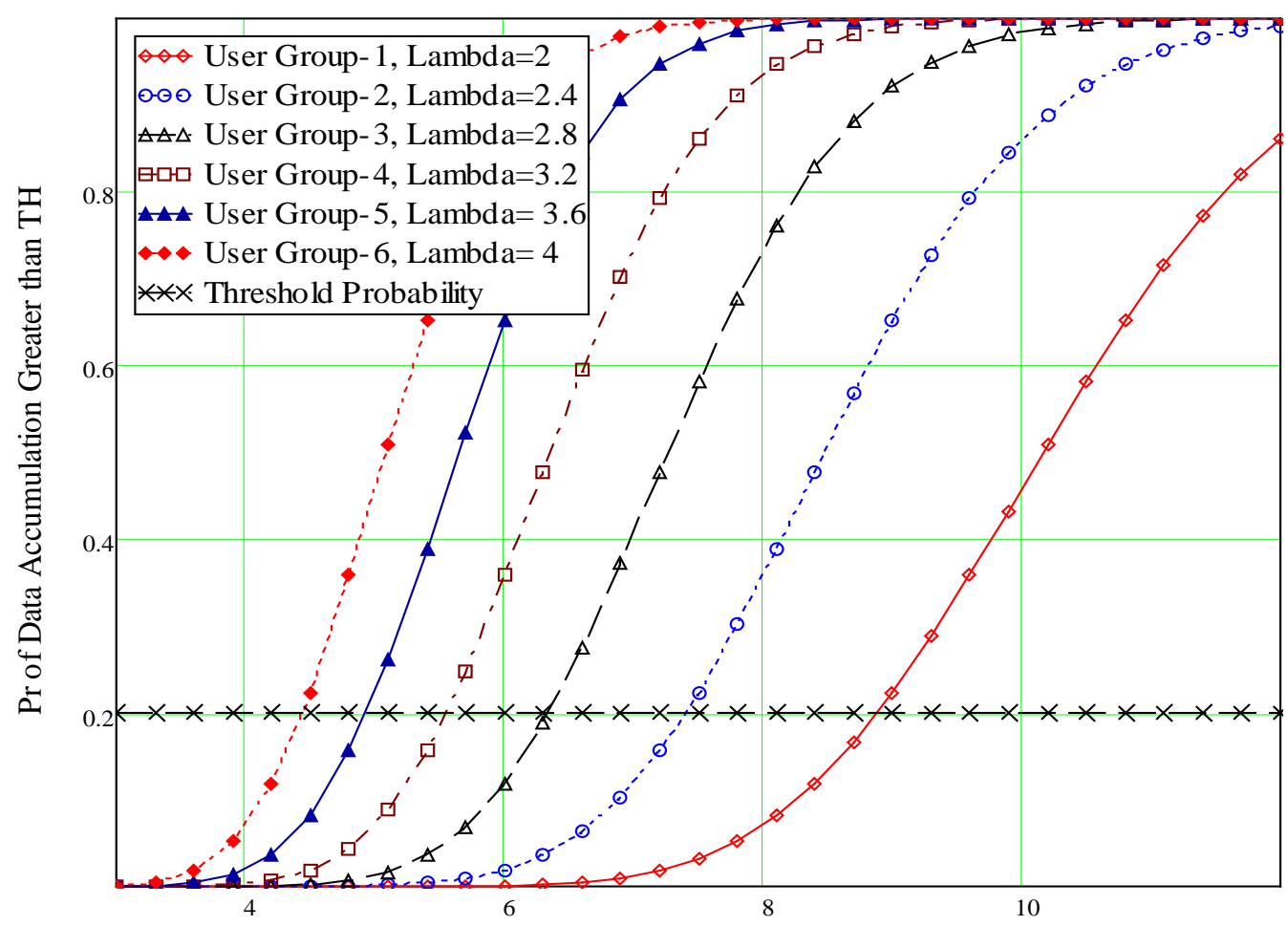

K

Figure 6. Variation of probability of data accumulation at higher arrival rate.

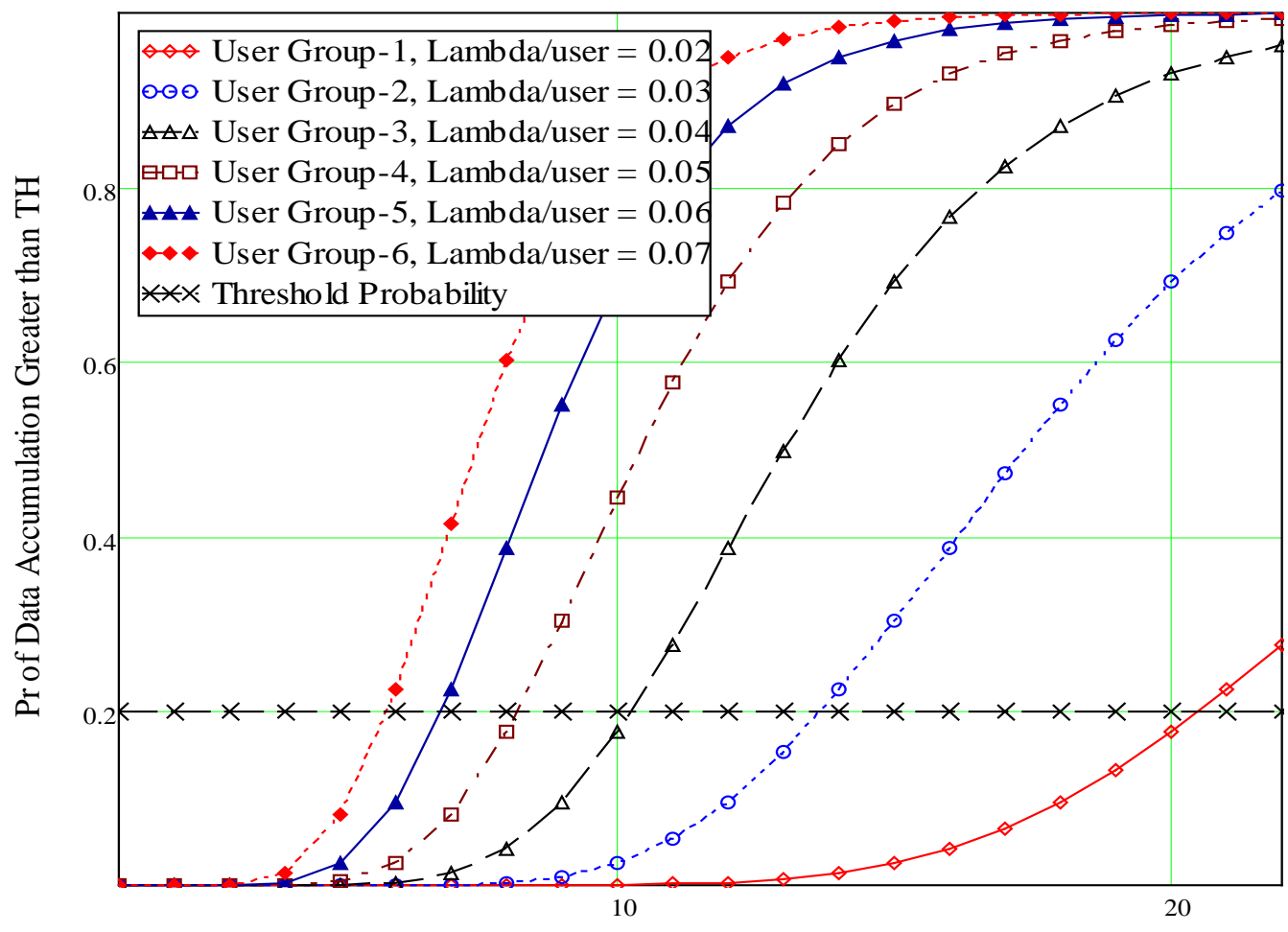

K

Figure 7. Variation of probability of data accumulation at lower arrival rate. 


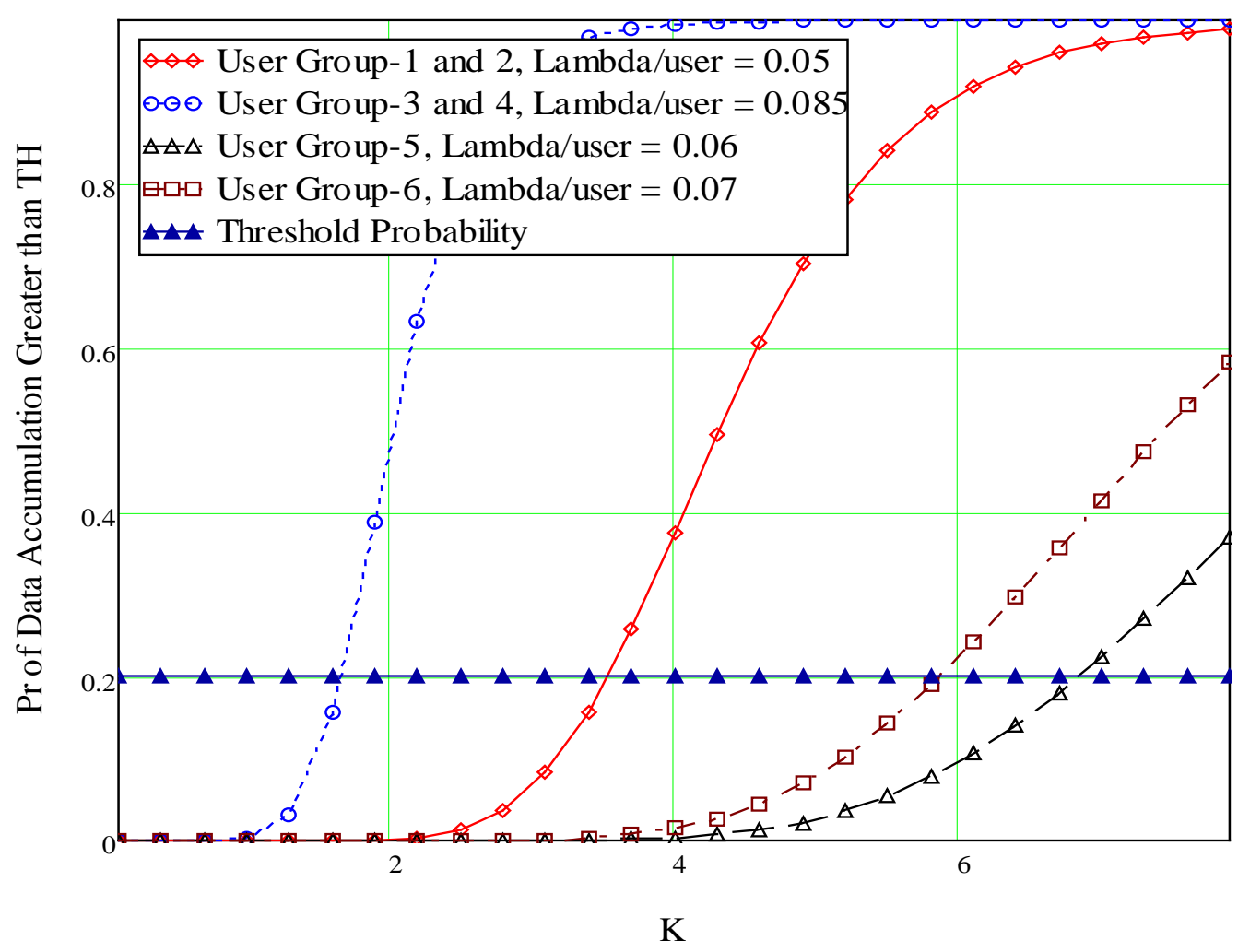

Figure 8. Combining group 1 and 2; 3 and 4.

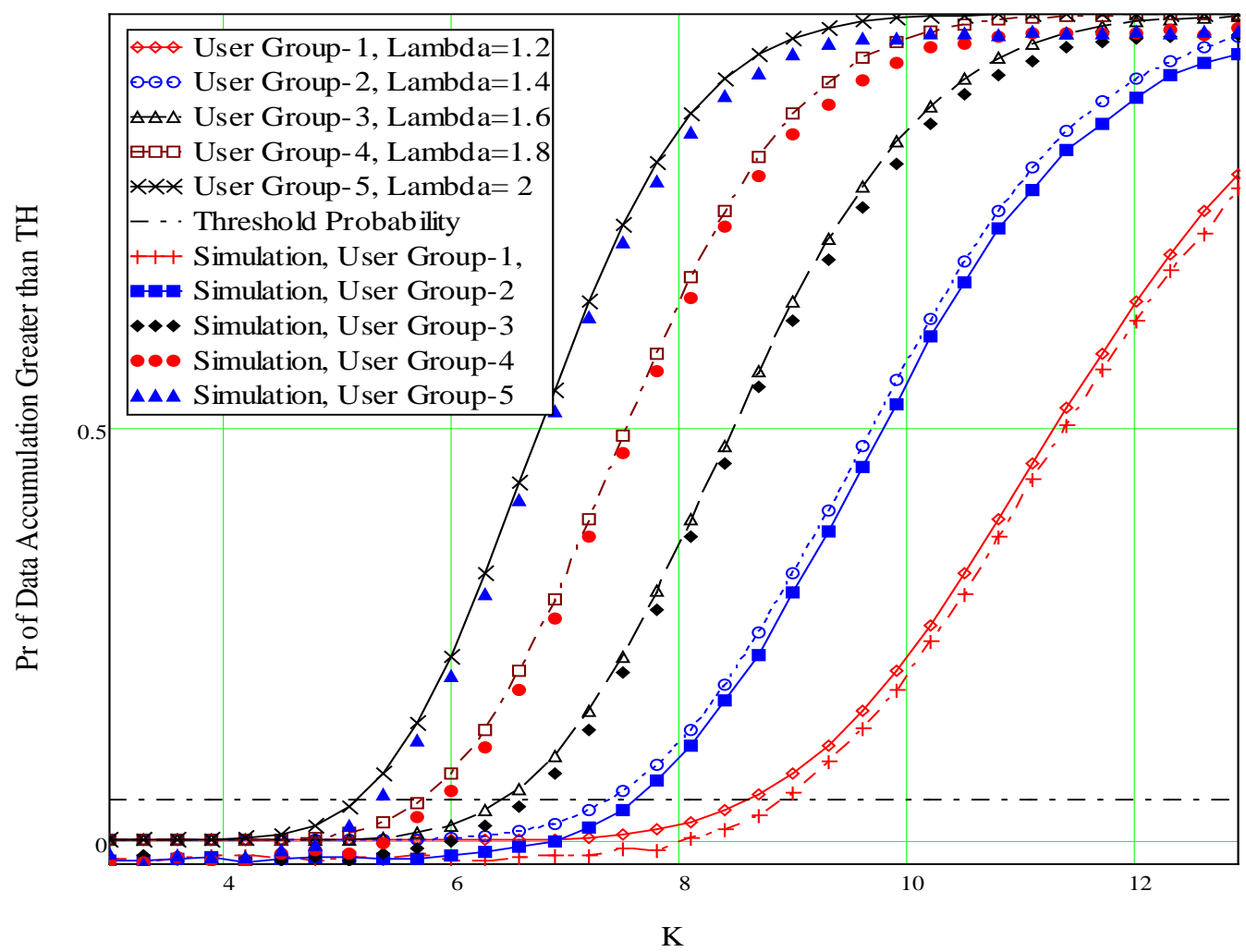

Figure 9. Comparison of analytical and simulation results. 
instead of $\mathrm{M} / \mathrm{M} / \mathrm{n} / \mathrm{K}$ has been considered. In near future, the similar analysis on $5 \mathrm{G}$ wireless network will be carried out. The research will also be extended in real system or with other simulation tools such as NS-3 or OPNET.

\section{References}

[1] Yang, C.-C., Chen, J.-Y., Mai, Y.-T. and Liu, H.-H. (2016) Integrated Power Saving for Relay Node and User Equipment in LTE-A. International Journal of Communication Systems, 29, 1342-1364.

[2] Nguyen, V.C., Pham, V.T. and Moon, B.-K. (2011) A New Energy Saving Mechanism in IEEE 802.16e/m. International Journal of Energy, Information and Communications, 2, 157-168.

[3] Bhaskaran, A.M. (2013) Energy Efficient Communication for IEEE 802.16e/m Networks. International Journal of Emerging Technology and Advanced Engineering, 3, 428-432.

[4] Chen, J.-S.R., Chang, Y.C. and Mei, H. (2015) Enhanced Power Saving Mechanism to Maximize Unavailability Interval in the IEEE 802.16 Mobile WiMAX. WSEAS Transactions on Communications, 14, 82-88.

[5] Gaikwad, V., Sharma, M. and Wagh, T. (2015) DTX Mechanism at Evolve Node for Efficient Power Saving in LTE Network. International Journal of Wireless and Microwave Technologies, 5, 47-55. http://dx.doi.org/10.5815/ijwmt.2015.05.05

[6] Liang, J.M., Hsieh, P.C., Chen, J.J. and Tseng, Y.C. (2013) Energy-Efficient DRX Scheduling for Multicast Transmissions in 3GPP LTE-Advanced Wireless Networks. 2013 IEEE Wireless Communications and Networking Conference (WCNC), Shanghai, April 2013, 551-556.

[7] Yang, C.C., Mai, Y.T., Chen, J.Y. and Kuo, Y.C. (2012) Integrated Load-Based Power Saving for BS and MSS in the IEEE 802.16 e Network. International Journal of Future Computer and Communication, 1, 83. http://dx.doi.org/10.7763/IJFCC.2012.V1.22

[8] Yang, C.C., Chen, J.Y., Mai, Y.T. and Liang, C.H. (2015) Adaptive Load-Based and Channel-Aware Power Saving for Non-Real-Time Traffic in LTE. EURASIP Journal on Wireless Communications and Networking, 2015, 215. http://dx.doi.org/10.1186/s13638-015-0435-x

[9] Chen, J., Tarn, W.H. and Lee, J.D. (2014) A Downlink and Uplink Alignment Scheme for Power Saving in IEEE802.16 Protocol. The Scientific World Journal, 2014, Article ID: 217973. http://dx.doi.org/10.1155/2014/217973

[10] Głąbowski, M. (2008) Modeling Systems with Multi-Service Overflow Erlang and Engset Traffic Streams. International Journal on Advances in Telecommunications, 1, 14-26.

[11] Veerarajan, T. (2003) Probability, Statistics and Random Process. Chap. 9, Tata McGraw-Hill Publishing Company Limited, New Delhi, 468-516.

[12] Ross, S.M. (2001) Introduction to Probability Models. 7th Edition, Academic Press, San Diego, USA, 216-225.

[13] Islam, I., Das, J.K. and Hossain, S. (2007) Modeling of Mixed Traffic for Mobile Cellular Network. Journal of Telecommunications and Information Technology, $\mathrm{nr}$ 1, 83-89.

[14] Morley, G.D. and Grover, W.D. (2000) Strategies to Maximize Carried Traffic in Dual-Mode Cellular Systems. IEEE Transactions on Vehicular Technology, 49, 357-366. http://dx.doi.org/10.1109/25.832966

[15] Islam, M.I., Rabbi, M.F., Khan, R.T. and Akhter, J. (2015) Performance Evaluation of Cognitive Radio Network Based on 2-D Markov Chain. Journal of Telecommunications and Information Technology, No. 3, 39.

[16] Hossain, M.A., Islam, M.I. and Amin, M.R. (2015) Analysis of Sleep Window of IEEE802.16m Network Based on State Transition Chain. Journal of Computer and Communications, 3, 77. http://dx.doi.org/10.4236/jcc.2015.312007

[17] Zhou, K., Nikaein, N. and Spyropoulos, T. (2013) LTE/LTE-A Discontinuous Reception Modeling for Machine Type Communications. IEEE Transactions on Wireless Communications Letters, 2, 102-105. 